\title{
The Shoulder Gradient in Patients with Unilateral Shoulder Impingement Syndrome
}

\author{
Hee-Sang Kim, M.D., Jong Ha Lee, M.D., Dong Hwan Yun, M.D., Jee-Sang Yun, M.D., \\ Yong Won Shin, M.D., Jinmann Chon, M.D., Dae Gyu Hwang, M.D.
}

Department of Physical Medicine and Rehabilitation, Kyung Hee University College of Medicine, Seoul 130-702, Korea

\begin{abstract}
Objective To investigate the relationship between the shoulder gradient and acromiohumeral interval of both shoulders in patients with unilateral shoulder impingement syndrome.

Method Using the angulometer, we measured the shoulder gradient in patients with unilateral shoulder impingement syndrome in a standing position. Using the radiography, we measured the acromiohumeral interval and the angle between a vertical line and a line connecting a superior angle with an inferior angle of the scapula.

Results In patients with unilateral shoulder impingement syndrome, the frequency of shoulder impingement syndrome was $76.2 \%$ ( 16 of 21 ) on the side of the relatively lower shoulder. The mean acromiohumeral interval on the side of the lower shoulder was $10.03 \pm 1.28 \mathrm{~mm}$, compared with $10.46 \pm 1.50 \mathrm{~mm}$ for the higher shoulder. The angle between a vertical line and a line connecting a superior angle with an inferior angle of the scapular of the side of the lower shoulder was $-0.31 \pm 3.73$ degrees, compared with $3.85 \pm 4.42$ degrees for the higher shoulder.

Conclusion The frequency of shoulder impingement syndrome was significantly higher on the side of the relatively lower shoulder, and there is no significant difference in the acromiohumeral interval between the side of the lower shoulder and that of the higher shoulder. In patients with unilateral shoulder impingement syndrome, the scapular on the side of lower shoulder was more rotated downward than on the side of the higher shoulder.
\end{abstract}

Key Words Shoulder impingement syndrome, Shoulder gradient, Acromiohumeral interval

\section{INTRODUCTION}

Endogenous, exogenous, and complex mechanisms have been presented as etiologic factors for shoulder

Received June 28, 2010; Accepted August 24, 2011

Corresponding author: Dae Gyu Hwang

Department of Physical Medicine and Rehabilitation, Kyung Hee University College of Medicine, 1, Hoegi-dong, Dongdaemun-gu, Seoul 130-702, Korea

Tel: +82-2-958-8565, Fax: +82-2-958-8560, E-mail: daegyuhwang@gmail. com

(c) This is an open-access article distributed under the terms of the Creative Commons Attribution Non-Commercial License (http:// creativecommons.org/licenses/by-nc/3.0) which permits unrestricted noncommercial use, distribution, and reproduction in any medium, provided the original work is properly cited.

Copyright () 2011 by Korean Academy of Rehabilitation Medicine impingement syndrome. According to Ogata and Unthoff, ${ }^{1}$ the endogenous mechanism starts with aging, circulatory disease, and differences in tensile stress within the rotator cuff muscles, causing degenerative change and damage to the tendon of the supraspinatus muscle. They also stated that damage of the rotator cuff muscles is due to degenerative changes in the tendon rather than by any collision and that it has no correlation with a degenerative change in the acromion.

Neer ${ }^{2}$ hypothesised that the exogenous mechanism involves the rotator cuff muscles, subacromial bursa and the long head of the biceps brachii muscle colliding with the anterior portion of the acromion and the related coracoacromial arch; this results in a disorder 
of the normal sliding function, causing continuous trauma every time the arm is raised and a progression to subacromial bursitis, biceps syndesmitis, and eventually inflammation and even rupture of the rotator cuff muscles.

In short, the exogenous mechanism of this thesis supports the theory that the shape of the acromion, the subacromial space, and the gradient of the acromion are correlated with damage to the rotator cuff muscles.

Also, Schamberger ${ }^{3}$ states that when the spine is bent laterally, the concave side of an intercostal space is narrowed, and this causes a depression of the shoulder joint and the scapula on the concave side. If the thoracic curvature bulges to the left, the right scapula is shown to be in retraction, depression, and downward rotation, and the facet of the glenoid fossa faces the posteroinferior side. When the slope of the glenoid shelf faces downwards, the passive support for the humerus is weakened, the capsule and rotator cuff muscles receive a great deal of traction force due to gravity, and this could be an etiologic factor in the development of supraspinatus tendinitis and other stages of the shoulder impingement syndrome.

On the basis of this study, we can therefore predict that a depression of the shoulder to the right by continuous sidewise curving of the spine may cause shoulder impingement syndrome in the right shoulder. Generally, if one compares the height of both shoulders in the standing position, most people have slight differences in the shoulder gradient. We therefore decided to investigate the relationships between the prevalence of shoulder impingement syndrome, shoulder gradient, acromiohumeral interval and location of the scapula.

\section{MATERIALS AND METHODS}

\section{Subjects}

The subjects were selected during the period April November, 2009, from those attending the Department of Rehabilitation Medicine, who complained of pain in the shoulder and upper extremities and were diagnosed as suffering from unilateral shoulder impingement syndrome on the basis of their history and a physical examination. Patients, who were experiencing pain in the shoulder and upper extremities through trauma, fracture, bone deformities, calcification, or shoulder joint instabilities, were excluded. All patients with a restriction in their range of motion during passive joint movement were also excluded. The patients selected as candidates for this study were those, who had positive results in all three of the following physical examinations: 1) the positive painful arc sign, which causes pain at a certain angle during flexion and abduction of the shoulder joint and then loss of the pain when the angle is increased, 2) the Neer and Hawkins-Kennedy tests, 3) pain induced in the resisted isometric external rotation, abduction and elbow extension test or pain in the Jobe test. A thorough examination of the contralateral shoulder was performed to rule out any patients with bilateral shoulder impingement syndrome.

Patients diagnosed with unilateral shoulder impingement syndrome, who had rheumatism, a systemic inflammatory disease, peripheral neuropathy, a history of stroke, prior surgery in the shoulder joint or cervical vertebrae, pain in the cervical vertebrae or restriction in the movement of the cervicothoracic vertebrae, spinal disease such as scoliosis, or adhesive capsulitis, were also
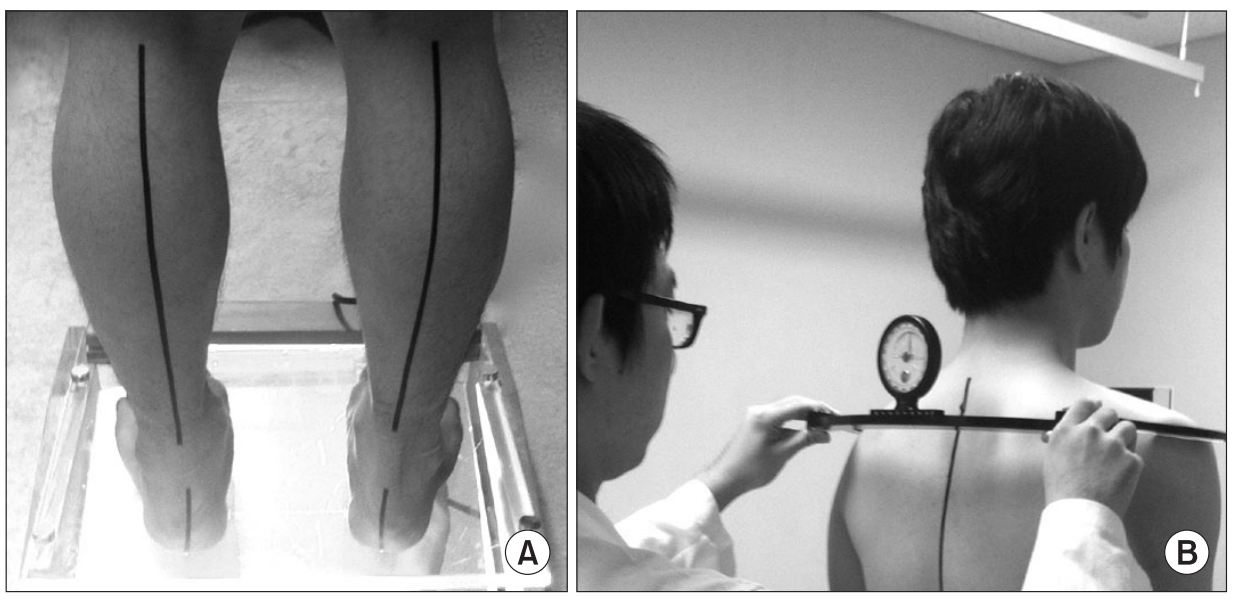

Fig. 1. Patient stood on the scaled podoscopesith ankles in neutral positionand knees are full extended (A). Shoulder gradoent was measured byangulmeter with each side of blade on patient's most lateral part of acromion (B). 
rejected as study participants.

\section{Methods}

The following measurements were made on each of the 21 patients finally selected as study subjects. The shoulder gradients were obtained by having each subject stand on an angulometer with feet spread $10 \mathrm{~cm}$ apart between both of the medial malleoli, and their heels were placed in a straight line. The ankles were fixed in a neutral

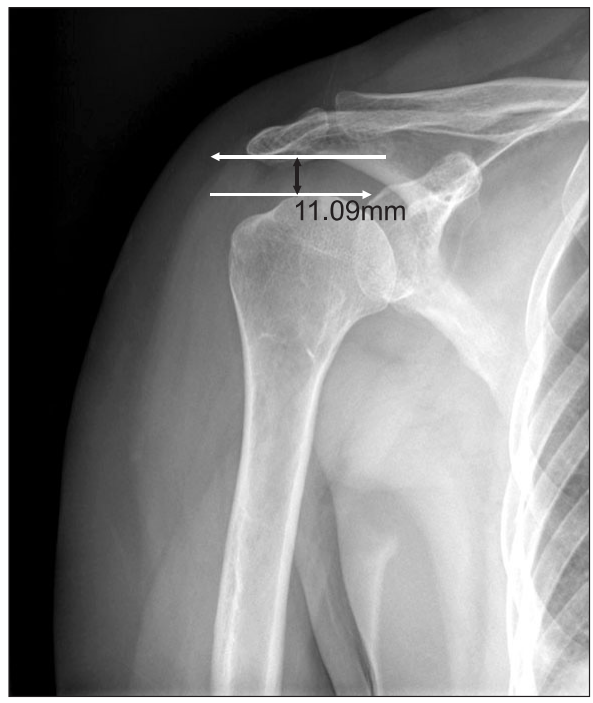

Fig. 2. This AP radiograph shows the acromiohumeral interval, which was measured as the shortest distance from the inferior surface of the acromion to the superior aspect of the humerus. position, and the knees completely extended. The relative heights of the shoulders were assessed by measuring the difference between the angles formed by both blades of the angulometer being placed on the upper, lateral side of the acromion of each shoulder (Fig. 1).

The acromiohumeral intervals were obtained from anteroposterior radiographs of both shoulders (Fig. 2). The rotation of the scapula was also obtained from the radiographs and involved measuring the angle formed by the vertical line and the line connecting the superior angle and the inferior angle. The angle was given a positive value if the scapula was rotated upwards (Fig. 3-A) and a negative value if the scapula was rotated downwards (Fig. $3-B)$. The average of three measurements was used.

\section{Statistical analysis}

The data were analyzed with SPSS for Windows (version 13.0). Differences between higher and lower shoulders and between shoulders affected or unaffected by impingement were compared in terms of acromiohumeral intervals and angles of scapular rotation using paired t-tests. Statistical significance was defined as a $p$-value $\leq 0.05$.

\section{RESULTS}

\section{Subject characteristics}

The 21 subjects ( 6 males and 15 females) were all righthanded, and their average age was 37.7 years (Table 1 ).
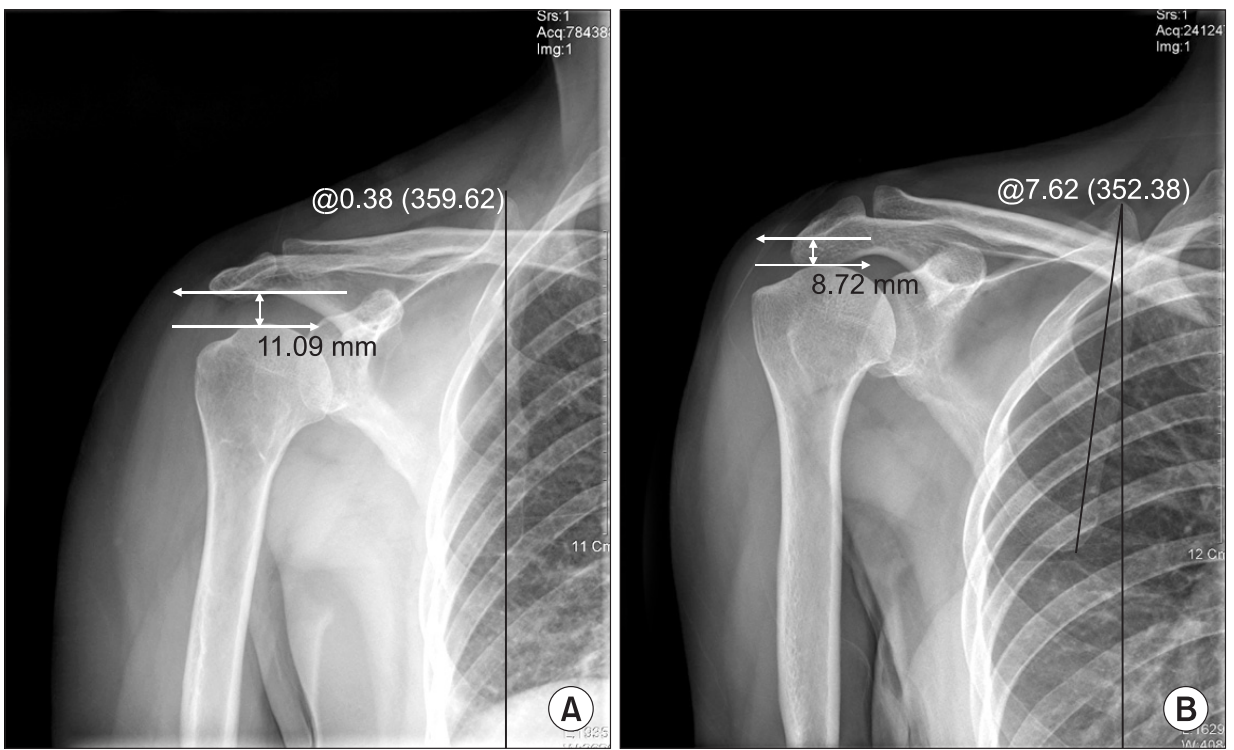

Fig. 3. This AP radiograph shows upward and downward rotation of scapula, which was measured as the angle between the vertical line and the line connecting a superior angle with an inferior angle of the scapula. Upward rotation of scapula (A), downward rotation of scapula (B). 
Prevalence of shoulder impingement syndrome in relation to the shoulder gradient

Thirteen subjects were affected with shoulder impingement syndrome in the right shoulder, and eight subjects were affected in the left. Whereas 16 subjects were affected in the relatively lower level shoulder, only

Table 1. General Characteristics of Subjects

\begin{tabular}{lc}
\multicolumn{1}{c}{ Characteristics } & Number/Value \\
\hline Gender & \\
Male/Female & $6 / 15$ \\
Duration of symptom (months) & $3.85 \pm 1.82^{*}$ \\
Age (years) & $37.67 \pm 11.76^{*}$ \\
\hline
\end{tabular}

*Values are mean \pm standard deviation

Table 2. Frequency and Site of Shoulder Impingement Syndrome

\begin{tabular}{lc}
\hline \multicolumn{1}{c}{ Characteristics } & Number \\
\hline Lower site & \\
Right/Left & $16 / 5$ \\
Impingement site & \\
Right/Left & $13 / 8$ \\
Comparison lower site with & \\
impingement site & \\
Agreement & $16(\mathrm{Rt}: 12 / \mathrm{Lt}: 4)$ \\
Disagreement & $5($ Rt.:1/Lt.:4) \\
\hline
\end{tabular}

five people were affected in the relatively higher level shoulder (Table 2). This observed relationship between the prevalence of impingement syndrome and the relative height of the affected shoulder was statistically significant (Fisher's exact probability test, $\mathrm{p}<0.05$ ).

Relationship between the acromiohumeral interval and shoulder gradient

While the mean acromiohumeral interval in the relatively lower shoulder was $10.03 \pm 1.28 \mathrm{~mm}$, the mean in the relatively higher shoulder was $10.46 \pm 1.50 \mathrm{~mm}$, but the difference was not statistically significant. Similarly, while the mean Acromiohumeral interval of the affected shoulder was $9.98 \pm 1.29 \mathrm{~mm}$, the Acromiohumeral interval of the unaffected shoulder was $10.51 \pm 1.48$ $\mathrm{mm}$, but again this difference failed to reach statistical significance (Table 3 ).

\section{Rotation of the scapula and shoulder gradient}

The mean rotational angle of $-0.31 \pm 3.73$ degrees in the relatively lower shoulder was significantly less $(\mathrm{p}<0.05)$ than the mean of $3.85 \pm 4.42$ degrees in the relatively higher shoulder. Also, the shoulders affected by impingement syndrome had a mean angle of $0.00 \pm 3.72$ degrees, which was significantly less $(\mathrm{p}<0.05)$ than the mean angle of unaffected shoulders $(3.54 \pm 4.71$ degrees) (Table 4).

Table 3. Acromiohumeral Intervals, which was Measured as the Shortest Distance from the Inferior Surface of the Acromion to the Superior Aspect of the Humerus

\begin{tabular}{lcccc}
\hline & Lower shoulder & Higher shoulder & Affected & Unaffected \\
\hline Acromiohumeral interval $(\mathrm{mm})$ & $10.03 \pm 1.28^{*}$ & $10.46 \pm 1.50^{*}$ & $9.98 \pm 1.29^{*}$ & $10.51 \pm 1.48^{*}$ \\
p-value & & 0.23 & & 0.13 \\
\hline
\end{tabular}

* Values are mean \pm standard deviation

Affected: Shoulder with impingement, Unaffected: Asymptomatic contralateral shoulder

Table 4. Upward, Downward Rotation of Scapula, which was Measured as the Angle between the Vertical Line and the Line Connecting a Superior Angle with an Inferior Angle of the Scapula

\begin{tabular}{|c|c|c|c|c|}
\hline & Lower shoulder & Higher shoulder & Affected & Unaffected \\
\hline Scapular angle $\left({ }^{\circ}\right)$ & $-0.31 \pm 3.73^{*}$ & $3.85 \pm 4.42^{*}$ & $0.00 \pm 3.72^{*}$ & $3.54 \pm 4.71^{*}$ \\
\hline p-value & \multicolumn{2}{|c|}{$<0.05$} & \multicolumn{2}{|c|}{$<0.05$} \\
\hline
\end{tabular}

*Values are mean \pm standard deviation

Affected: Shoulder with impingement, Unaffected: Asymptomatic contralateral shoulder 


\section{DISCUSSION}

This study determined the relationships between the shoulder gradient and the site and prevalence of shoulder impingement syndrome, the Acromiohumeral interval, and level of the scapula. The prevalence of unilateral shoulder impingement syndrome was higher in the relatively lower level shoulder, and the scapula of the lower shoulder was significantly rotated downwards compared with the contralateral one. However, the relative levels of the right and left shoulders were not significantly associated with subscapular distance.

Schamberger ${ }^{3}$ stated that, when the spine is laterally bent, the concave side of the intercostal space is narrowed, and the scapula of the concave side undergoes retraction, depression, and downward rotation. This results in the facet of the glenoid fossa facing posteroinferiorly and the slope of the glenoid shelf pointing downwards. The passive support of the humerus is weakened, the capsule and rotator cuff muscles receive considerable traction force due to gravity, and the consequence may be supraspinatus tendinitis or shoulder impingement syndrome. The theory that continually bending the spine sideways, which lowers the shoulder to the right, could cause shoulder impingement syndrome in the right shoulder joint was supported in the present study by the higher frequency of shoulder impingement syndrome in the relatively lower shoulder when both shoulder levels were compared in unilaterally affected patients. Likewise in our study, the scapula of the lower shoulder was relatively rotated downward.

A shortening of the Acromiohumeral interval, which provides the space for movements of the rotator cuff muscles, will probably increase the risk of damage to the rotator cuff muscles. Radiologic studies have shown that supraspinatus rupture occurs if the Acromiohumeral interval is $6 \mathrm{~mm}$ or less, and that associated multiple muscle ruptures are highly likely if the distance is $7 \mathrm{~mm}$ or less. ${ }^{12}$ Park et al. ${ }^{13}$ reported that the Acromiohumeral interval measured using MRI was significantly reduced in the affected shoulder $(7.7 \mathrm{~mm})$ compared with that in the normal unaffected shoulder $(10.3 \mathrm{~mm})$. However, in our study, there was no statistical difference between the measurements of the Acromiohumeral interval in the affected $(9.98 \pm 1.29 \mathrm{~mm})$ and unaffected $(10.51 \pm 1.48 \mathrm{~mm})$ shoulders even though the Acromiohumeral interval of the affected shoulder was shorter compared with the unaffected side. This is probably due to our recruitment of comparatively young subjects with a mean age of 37.7 years and their short histories of shoulder impingement syndrome.

Shiri et al. ${ }^{17}$ found that there was a higher prevalence of biceps tendinitis and rotator cuff damage related to shoulder impingement syndrome in the dominant arm. In our study, 13 patients out of 21 patients, who were all right handed contracted shoulder impingement syndrome on the right side, but the difference was not quite as large as in the study by Shiri et al. However, 16 subjects of the 21 right handed patients were observed to have lower shoulder levels on the dominant side. Nevertheless, since our study did not include any left-dominant subjects, further studies are needed to investigate an association between shoulder gradient and the dominant hand.

The limitations of this study include the small sample size and a lack of evaluation of three-dimensional movements in the tilting or transverse rotation of the scapula. In addition, there is the need in future studies to take into account the many variables, which might cause differences in shoulder levels, such as the different lengths of the lower extremities and the level of the pelvic bone. Nevertheless, the findings of the present study suggest that an effective treatment for shoulder impingement syndrome may be found by correcting the factors responsible forincreasing shoulder gradients. Future studies should be directed towards evaluating the effectiveness of this approach.

\section{CONCLUSION}

In unilateral shoulder impingement syndrome, there was a higher prevalence in the relatively lower shoulder, and the scapula of the relatively lower shoulder showed statistically significant signs of downward rotation.

\section{REFERENCES}

1. Ogata S, Uhthoff HK. Acromial enthesopathy and rotator cuff tear. A radiologic and histologic postmortem investigation of the coracoacromial arch. Clin 
Orthop Relat Res 1990; 254: 39-48

2. Neer CS. Impingment lesions. Clin Orthop Relat Res 1983; 173: 70-77

3. Schamberger $\mathrm{W}$. The malalignment syndrome. In: Schamberger W, Editor. The malalignment syndrome, 1st ed, New York, Churchill Livingstone, 2002, 106-110

4. Shibuta H, Tamai K, Tabuchi K. Magnetic resonance imaging of the shoulder in abduction. Clin Orthop Relat Res 1998; 348: 107-113

5. Tirman PF, Steinbach LS, Belzer JP, Bost FW. A practical approach to imaging of the shoulder with emphasis on MR imaging. Orthop Clin North Am 1997; 28: 483-515

6. Allmann KH, Uhl M, Gufler H, Biebow N, Hauer MP, Kotter E, Reichelt A, Langer M. Cine-MR imaging of the shoulder. Acta Radiol 1997; 38: 1043-1046

7. Hijioka A, Suzuki K, Nakamura T, Hojo T. Degenerative change and rotator cuff tears. An anatomical study in 160 shoulders of 80 cadavers. Arch Orthop Trauma Surg 1993; 112: 61-64

8. Schneeberger AG, Nyffeler RW, Gerber C. Structural changes of the rotator cuff caused by experimental subacromial impingement in the rat. J Shoulder Elbow Surg 1998; 7: 375-380

9. Rathbun JB, Macnab I. The microvascular pattern of the rotator cuff. J Bone Joint Surg Br 1970; 52: 540-553

10. Ozaki J, Fuzimoto S, Nakagawa Y, Masuhara K, Tamai $\mathrm{S}$. Tears of the rotator cuff of the shoulder associated with pathological changes in the acromion. A study in cadevara. J Bone Joint Surg Am 1988; 70: 1224-1230

11. Petersson CJ, Redlund-Johnell I. The subacromial space in normal radiographs. Acta Orthop Scand 1984; 55: 57-58

12. Norwood LA, Barrack R, Jacobson KE. Clinical presentation of complete tears of the rotator cuff. J Bone Joint Surg Am 1989; 71: 499-505

13. Park TS, Rha JD, Kim YH, Yang KH, Choi SM. A study of the measurement of the distance of the subacromial space in normal shoulder. J Korean Sport Med 1994; 12: $103-108$

14. Brossmann J, Preidler KW, Pedowitz RA, White LM, Trudell D, Resnick D. Shoulder impingement syndrome: influence of shoulder position on rotator cuff impingement-an anatomic study. Am J Roentgenol 1996; 167: 1511-1515

15. Solem-Bertoft E, Thuomas KA, Westerberg CE. The influence of scapular retraction and protraction on the width of the subacromial space. An MRI study. Clin Orthop Relat Res 1993; 296: 99-103

16. Nove-Josserand L, Edwards TB, O'Connor DP, Walch G. The acromiohumeral and coracohumeral intervals are abnormal in rotator cuff tears with muscular fatty degeneration. Clin Orthop Relat Res 2005; 433: 90-96

17. Shiri R, Varonen H, Heliövaara M, Viikari-Juntura E. Hand dominance in upper extremity musculoskeletal disorders. J Rheumatol 2007; 34: 1076-1082 